\title{
Leprosy of the eustachian tube (nasopharyngoscopic study)
}

\author{
N. K. SONI \\ Department of Ear, Nose \& Throat, Sardar Patel Medical College \\ and Associated Group of Hospitals, Bikaner (Rajasthan) India
}

Accepted for publication 27 June 1995

Summary The technique of nasopharynogoscopy affords an accurate assessment of the lesions at the orifices of the eustachian tube. It was performed in 30 patients suffering from lepromatous leprosy in order to determine the type, nature and site of the lesion. Involvement of the eustachian tube in leprosy may begin with a localized area of erythema progressing to granuloma formation or ulceration. Leprous lesion at the eustachian tube orifices was related with subsequent changes in the tympanogram pattern. Nasopharyngoscopy is also found to be of therapeutic value in removing the crust, discharge and granulations at the eustachian tube orifices.

\section{Introduction}

Leprosy is a systemic disease which involves the upper respiratory tract, including the nasal mucosa as well as skin and peripheral nerves. In lepromatous leprosy, over $95 \%$ of patients have involvement of nasal mucosa. ${ }^{1,2}$ The disease extends and involves the entire nasal cavity up to and including the posterior part and the nasopharynx. The orifice of the eustachian tube is situated on the lateral wall of the nasopharynx. Because of nasal and nasopharyngeal involvement there is a possibility of the spread of disease into the eustachian tube.

An extensive survey of the literature reveals no study of direct visualization of the eustachian tube in patients suffering from lepromatous leprosy.

Pure tone audiometry and tympanometry are of limited help in assessing whether middle-ear problems are due to actual involvement of the eustachian tubes with leprosy or to the secondary effects of nasal leprosy.

Nasopharyngoscopy is the only method which can give accurate information in respect to the type, nature and site of lepromatous lesions at the nasopharyngeal orifice of the eustachian tube. In view of the high incidence of nasal involvement associated with a highly infectious discharge and the possibility of the spread of leprosy into the

Correspondence: Dr N. K. Soni, H-6 PBM Hospital Campus, Bikaner 334003 (Raj), India 
Table 1. Showing clinical classification of stages in intranasal leprosy

\begin{tabular}{|c|c|c|}
\hline Stage & Finding & $\begin{array}{c}\text { No. of } \\
\text { patients }\end{array}$ \\
\hline Stage I (invasive) & Congestion, nasal discharge, early atrophic area. & 5 \\
\hline Stage II (proliferative) & $\begin{array}{l}\text { Polypoidal proliferation grey nodule, congestion oedema } \\
\text { or early ulceration. }\end{array}$ & 12 \\
\hline Stage III (destructive) & $\begin{array}{l}\text { Ulcerative lesion septal perforation thick nasal discharge } \\
\text { and crusting. }\end{array}$ & 10 \\
\hline Stage IV (resolution) & Stage of resolution fibrosis and scarring. & 3 \\
\hline
\end{tabular}

eustachian tube, it was decided to investigate the eustachian tube by nasopharyngoscopy.

\section{Material and methods}

Thirty patients with bacillary positive untreated lepromatous leprosy were taken for the present study. Their ages ranged from 15 to 55 years. There were 19 males and 11 females.

\section{Results}

In each case, a complete ENT examination was performed. Intranasal examination was done and findings were recorded and categorized into different stages according to the classification used by Job et al. ${ }^{3}$ as shown in Table 1.

Complete otoscopic examination was done in each case and wax removed if present and the findings were recorded. Seven patients $(23 \%)$ showed clinical findings in one or both ears, of retracted tympanic membranes or secretory otitis media (Table 2). Similarly pharyngeal and laryngeal examinations were done in each case. The details have already been reported. ${ }^{4}$ Pure tone audiometry was performed in each case. The details have been described elsewhere. Tympanometry was performed using an American Electromedics 86 AR tympanometer over a pressure range -400 to $+200 \alpha \mathrm{Pa}$. The details of the results have already been reported. ${ }^{5-7}$

X-rays of the nose and paranasal sinuses (Water's view) and lateral view of the nasopharynx were taken. The X-ray findings in the paranasal sinuses revealed that the

Table 2. Showing otoscopic finding in lepromatous leprosy

\begin{tabular}{lr}
\hline Finding & Number of patients \\
\hline Retracted ear drum & 4 patients: Both ear: 2 \\
One ear: 2 \\
Secretory otitis media & 3 Patients: Both ear: 1 \\
One ear: 2
\end{tabular}


Table 3. Various types of leprosy lesion seen in nasopharyngoscopy

\begin{tabular}{lc}
\hline Types of lesion & No. of cases \\
\hline Normal & 21 \\
Superficial inflammation with or without ulcer & 2 \\
Ulcers of variable size and thick secretion deposit & 3 \\
Granulomatous lesion & 3 \\
Healed ulceration with scar & 1 \\
\hline Total & 30 \\
\hline
\end{tabular}

maxillary antum was found to be affected more commonly, i.e. $60 \%$ more than the others (ethmoidal $33 \cdot 33 \%$ and frontal sinuses $30 \%$ ). There was evidence of diffuse mucosal thickening, patchy mucosal thickening to complete opacity of the sinuses. The details of the findings have already been reported elsewhere. ${ }^{8}$

All the cases underwent nasopharyngoscopy using a Storz $90^{\circ}$ angled rigid nasopharyngoscope to study the eustachian tube orifices. The type, nature and site of the pathology observed was recorded. Biopsy was taken in 8 patients which revealed the typical picture of lepromatous leprosy with positive acid-fast bacilli (AFB).

Nine out of the 30 cases revealed pathological changes around the pharyngeal ends of the eustachian tubes while the remaining cases were normal in nasopharyngoscopy. The various types of pathology seen are shown in Table 3. The eustachian tube orifices are more commonly affected in stage III (ulcerative lesions, septal perforation with thick nasal discharge or crusting) and stage II (polypoidal proliferation greyish node, congestion, oedema or early ulceration) forms of intranasal leprosy (Table 4).

\section{Discussion}

Nasopharyngoscopy is the most appropriate technique for making an accurate diagnosis of lesions at the orifices of the eustachian tube. This technique has repeatedly been shown to provide information which is superior to that obtained by clinical or radiological investigations. Again, pure tone audiometry and tympanometry are of limited value in assessing whether middle-ear problems are due to actual involvement of the eustachian tubes with leprosy or to secondary affects of an extensive intranasal

Table 4. Relationship between clinical staging of intranasal leprosy and lesions at the eustachian tube orifices

\begin{tabular}{llccc}
\hline & & \multicolumn{2}{c}{ Eustachian tube lesion } \\
\cline { 3 - 5 } Stage of nasal leprosy & $\begin{array}{c}\text { Total No. of } \\
\text { patients }\end{array}$ & Normal & Positive \\
\hline I & Invasive & 5 & 5 & - \\
II & Proliferative & 12 & $\mathbf{8}$ & 4 \\
III & Destructive and ulceration & 10 & $\mathbf{6}$ & 4 \\
IV & Resolution and fibrosis & 3 & 2 & 1 \\
& & & & \\
\hline
\end{tabular}


leprosy. Nasopharyngoscopy not only allows direct visual assessment of the pathology but also allows biopsies to be taken from the lesion and thus confirms the lesion either to be actual leprosy or that the pathology is due to the secondary effects of nasal leprosy. Nasopharyngoscopy in leprosy is not only a diagnostic procedure but also has a therapeutic value. Collected secretions, crusting, and granulations can all be removed effectively from the orifice of the eustachian tube under direct vision with subsequent symptomatic improvement. It may also prove helpful in an accurate follow up of cases to assess the benefit of planned therapy.

The nasopharyngoscopy in the present 30 lepromatous leprosy patients revealed the presence of positive lesions at the orifices of the eustachian tube in 9 cases.

Similar to the intranasal lesion, different types of leprosy lesions were found at the pharyngeal orifice of the eustachian tube. Leprous involvement may begin with a localized area of erythma, progressing to ulceration or localized granuloma formation depending upon the immunity of the host and virulence of the organism. Scarring or evidence of healing may be seen in places.

The microscopic pathology of the lesions at the orifices of the eustachian tube is similar to other leprous lesion. Biopsy of the non-ulcerative form was not performed because of the risk of converting an early mucosal lesion into an ulcerative form and the chance of enhancing the disease by direct contact of the lesion with an infected discharge.

During nasopharyngoscopy cleaning of the eustachian tube orifices by removing the crust, discharge or granulations resulted in improvement in audiometric and tympanometric findings. Thus it is suggested that periodic cleaning of eustachian tube orifices along with prolonged antileprosy therapy may be more beneficial in preventing permanent disability of the eustachian tube by scaring or stenosis, especially in resistant cases.

The present study reveals that $30 \%$ of the patients have a subclinical involvement of the eustachian tube which may be diagnosed only on nasopharyngoscopy. This early diagnosis is of utmost importance to allow specific treatment before permanent damage to the eustachian tube has occurred.

\section{References}

1 Barton RPE. A clinical study of the nose in lepromatous leprosy. Lepr Rev, 1974; 45: 135-40.

2 Barton RPE. Clinical manifestation of lepromatous rhinitis. Ann Otology, Rhinol, Lar yngol, 1976; 85: 74-82.

3 Job CK, Karat ABA, Karat S. The histopathological appearance of leprorhinitis with pathogenesis of septal perforation in leprosy. J Laryngol Otology, 1961; 80: 718-32.

4 Soni NK. Leprosy of the larynx. J. Laryngol Otolog, 1992; 106: 518-20.

5 Soni NK. Middle ear function in Hansen's disease (Tympanometric study). In proceedings of International Congress of Leprosy; Abstract published in Int J Lepr, 1989; (No.1 Suppl.) 57: 495.

6 Soni NK. Antroscopic study of the maxillary antrum in lepromatous leprosy. J Laryngol Otology, 1989; 103: 502-3.

7 Soni NK. Eustachian tube functions in lepromatous leprosy. Ind J Lepr, 1994.

8 Soni NK. Radiological study of the paranasal sinuses in lepromatous leprosy. Ind J Lepr, 1988; 60: $285-9$. 